\title{
The influence of Apolipoprotein E genotype on regional pathology in Alzheimer's disease
}

\author{
Marwan N Sabbagh ${ }^{1 *}$, Michael Malek-Ahmadi ${ }^{1}$, Brittany N Dugger ${ }^{2}$, Katarina Lee ${ }^{1}$, Lucia I Sue ${ }^{2}$, Geidy Serrano²,
} Douglas G Walker ${ }^{3}$, Kathryn Davis ${ }^{1}$, Sandra A Jacobson ${ }^{1}$ and Thomas G Beach ${ }^{1,2}$

\begin{abstract}
Background: Carriers of the ApoE $\varepsilon 4$ allele are at a greater risk for developing Alzheimer's disease (AD) and those who do develop AD tend to have a much greater neuropathological disease burden. Although several studies have shown significant differences in AD pathology among $\varepsilon 4$ carriers and non-carriers, few have characterized these differences in terms of brain region and neuropathological score frequency.

Methods: 566 pathologically-confirmed AD cases who were followed prospectively with antemortem dementia diagnoses (312 ApoE $\varepsilon 4$ carriers and $254 \mathrm{ApoE} \varepsilon 4$ non-carriers) were compared on the frequencies of neuropathological frequency scores (none, sparse, moderate, frequent) among several different brain regions (frontal, temporal, parietal, hippocampal, and entorhinal) using the CERAD scoring system. Pathology score frequencies were analyzed by carrier status ( $\varepsilon 4$ carrier vs. $\varepsilon 4$ non-carrier) and by genotype $(2 / 3,3 / 3,2 / 4,3 / 4,4 / 4)$. Both analyses investigated pathology score frequencies among different brain regions (frontal, temporal, parietal, hippocampal, and entorhinal).
\end{abstract}

Results: $\varepsilon 4$ carriers had a significantly lower age at death $(p<0.001)$ and significantly higher Braak scores $(p<0.001)$ than $\varepsilon 4$ non-carriers. Genotype comparison revealed that plaque and tangle pathologies increased in the following pattern, $2 / 3<3 / 3<2 / 4<3 / 4<4 / 4$, for several brain regions. When stratified by age and ApoE $\varepsilon 4$ carrier status, $\varepsilon 4$ carriers tended to have significantly more frequent scores across most cortical areas. However, non-carriers age 90 and older tended to have greater plaque pathology than carriers. For tangle pathology, $\varepsilon 4$ carriers tended to have significantly more "frequent" scores than non-carriers, except for the hippocampal and entorhinal areas in individuals age 90 and older.

Conclusions: ApoE $\varepsilon 4$ carriers had a significantly higher percentage of "frequent" scores for plaques and tangles when compared to ApoE $\varepsilon 4$ non-carriers for several brain regions. However, $\varepsilon 4$ non-carriers age 90 and older tended to have less plaque and tangle pathology in certain brain regions. These results demonstrate that $A D$ pathology may manifest itself differently based on ApoE genotype and suggest that ApoE carriers and non-carriers may have different patterns of AD neuropathology location and density.

\section{Background}

It has been shown that the three main polymorphisms (E2, E3, E4), coded by the $\varepsilon 2$, $\varepsilon 3$, and $\varepsilon 4$ alleles on chromosome 19, of the apolipoprotein $\mathrm{E}$ (ApoE) gene result in differential risk factors for Alzheimer's disease (AD) for those who carry them [1-6]. The $\varepsilon 2$ isoform, the rarest allele out of the three [7], has demonstrated a protective effect, while subjects heterozygous for the $\varepsilon 4$

\footnotetext{
* Correspondence: marwan.sabbagh@bannerhealth.com

${ }^{1}$ The Cleo Robert Center for Clinical Research, Banner Sun Health Research Institute, Sun City, AZ, USA

Full list of author information is available at the end of the article
}

allele experience a three-fold increase in risk for sporadic $\mathrm{AD}$ and those homozygous for $\varepsilon 4$ allele have a 12-18 fold risk increase [5,7,8]. The $\varepsilon 4$ allele has also been shown to shift the age of onset of AD earlier than those without the allele [8]. Although the $\varepsilon 3$ allele is the most common allele [9], several studies have shown that the $\varepsilon 4$ allele is much more frequent among those with late-onset familial and sporadic AD in comparison to controls [10]. The $\varepsilon 3$ isoform displays an intermediate effect and, due to its higher frequency, its effect on pathologies is taken as the base line comparison for the $\varepsilon 2$ and $\varepsilon 4$ isoforms [7].

\section{Biomed Central}


In addition to being linked to $\mathrm{AD}, \mathrm{ApoE} \varepsilon 4$ has also been strongly linked to increased deposition of the main hallmark proteins in $\mathrm{AD}$ brains, amyloid beta $(\mathrm{A} \beta)$ and neurofibrillary tangles (NFTs), as well as correlating with certain biomarkers found in plasma and cerebrospinal fluid of $\mathrm{AD}[10,11]$. With respect to pathological staging schemes for $A \beta$ deposits and NFTs [12,13] $\varepsilon 4$ allele carriers tend to be more advanced implying earlier onset of $\mathrm{AD}$ [2]. Furthermore, the presence of ApoE $\varepsilon 4$ allele has been shown to correlate with increased deposition of both amyloid and tau pathology in the neocortex [1]. In non-demented subjects over age 60, ApoE $\varepsilon 4$ is associated with a higher burden of total parenchymal and vascular amyloid neuropathology than non-demented individuals, but with no difference in tau pathology [3]

The intent of this study is to determine how ApoE genotype affects $\mathrm{AD}$ pathology by brain region. By comparing the frequencies of neuropathological scores for several different brain regions, we aim to produce a more refined and specific characterization AD pathology in a large, clinicopathologically confirmed sample that is stratified by age and ApoE genotype.

\section{Methods}

\section{Subjects}

The subjects in this study were recruited from the Banner Sun Health Research Institute Brain and Body Donation Program located in Sun City, Arizona [14]. Individuals enter the program by voluntarily agreeing to brain autopsy after death; persons with dementia are signed into the program by their legal representative. All participants signed informed consent prior to enrolling into the program which was approved by the Banner Health Institutional Review Board (IRB).

Data from 775 cases autopsied between January of 1997 and December 2011 were available for the analysis. Only individuals who had received both a clinical diagnosis of dementia and a neuropathological diagnosis of AD were included in the study. 209 individuals were excluded due to incomplete neuropathological information, other clinical diagnoses of non-AD dementia or unavailable genotype.

The final sample size was 566 and consisted of 312 ADApoE4+ and 254 AD-ApoE4- cases who were followed prospectively with antemortem dementia diagnoses. The sample did not differ statistically with respect to numbers of males $(n=295)$ and females $(n=271)$. Clinical AD diagnoses were made using DSM-IV criteria [15].

\section{Pathological assessment}

Neuropathological AD diagnoses were made according to National Institute on Aging/Reagan Institute criteria [16] and included those categorized as "intermediate" or "high" probability.
Senile plaque and neurofibrillary tangle load scores were obtained using the CERAD scoring system with separate semi-quantitative density estimates of none, sparse, moderate, or frequent (converted to a 0-3 scale for statistical purposes) using standardized published templates [17]. Regions scored included cortical gray matter from frontal $(\mathrm{F})$, temporal $(\mathrm{T})$, parietal $(\mathrm{P})$, hippocampal CA1 $(\mathrm{H})$, and entorhinal (E) regions. The individual carrying out the scoring (TB) was blinded to demographics and ApoE status. Plaque and tangle scores from the F, T, P, H, and $\mathrm{E}$ were compared between ApoE $\varepsilon 4$ carriers and ApoE $\varepsilon 4$ non-carriers and also between each individual isoform $[12,13]$.

\section{ApoE genotyping}

DNA for ApoE genotyping from autopsy cases was extracted from pieces of fixed cerebellum tissue. Tissue (100 mg) was digested to completion with proteinase $\mathrm{K}$ $(1 \mathrm{mg} / \mathrm{ml})$ at $55^{\circ} \mathrm{C}$ and extracted with phenol/chloroform. DNA was recovered by isopropanol precipitation. For PCR reactions, $500 \mathrm{ng}$ of DNA from each sample was used. PCR primers, amplification conditions employed, and identification of ApoE genotypes by Hha I digestion of amplified material, were carried out according to a published protocol [18]. Digested fragments were separated by electrophoresis through $9 \%$ acrylamide gels and identified by staining with fluorescent dye Gel Red (Biotium, Hayward, CA).

\section{Statistical analysis}

For demographic data, chi-square analysis was used to assess the association between ApoE $\varepsilon 4$ carrier status and gender. The Mann-Whitney $\mathrm{U}$ test was used to discern differences on age at death and years of education between ApoE \&4 carriers and non-carriers. The effect size of these differences was assessed using Cohen's $d$.

For the pathological data, the sample was stratified by ApoE \&4 carrier status and age with the frequency of pathological score reported for each stratified group. The sample was divided into the following age groups: $\leq 69$, $70-79,80-89, \geq 90$. The data were also stratified by ApoE genotype to examine pathological differences between each ApoE isoform. For the stratified carrier status and age analyses, the Mann-Whitney $U$ test was used to compare plaque and tangle rating frequency differences between ApoE $\varepsilon 4$ carriers and non-carriers in each age group. For the genotype analyses, the Kruskal-Wallis test was used to compare differences of plaque and tangle pathology rating frequencies. Groupwise comparisons were carried out using the Dwass-Steel-Chritchlow-Fligner test.

\section{Results}

For the sample of 566, the average age at death was 82.56 (8.33) years and the average education level was 
14.54 (2.67) years. The sample was comprised of 295 males and 271 females. Demographic characteristics broken down by ApoE $\varepsilon 4$ carrier status are displayed in Table 1. There was no significant association between ApoE $\varepsilon 4$ carrier status and gender $\left(\chi^{2}=1.17, \mathrm{df}(1), \mathrm{p}=0.28\right)$. Education was not significantly different between carriers and non-carriers, however $\varepsilon 4$ carriers had a significantly younger age at death and significantly higher Braak score than non-carriers. The effect size for age at death was small, while the effect size for Braak score was moderate. Table 2 displays the demographic and clinical data for each ApoE genotype.

Scores for plaque and tangle frequencies stratified by age group and ApoE $\varepsilon 4$ carrier status are shown in Tables 3 and 4 . In the $\leq 69$ group, no statistically significant differences were found between ApoE $\varepsilon 4$ carriers and noncarriers for plaques and tangles in all cortical areas. Although the percentages of individuals show that ApoE $\varepsilon 4$ carriers had more "frequent" scores, the lack of statistical significance is likely due to the small cell counts for this age group. For the 70-79 age group, ApoE\&4 carriers had significantly more "frequent" plaque density scores than non-carriers in all cortical areas, except for the temporal area. For tangle density scores in the 70-79 age group, the only area to show statistically significant difference was the hippocampal area. In the 80-89 age group, ApoEع4 carriers had significantly more "frequent" plaque density scores than non-carriers in all cortical areas for both plaque and tangle densities. For the $\geq 90$ age group, non-carriers had significantly more "frequent" plaque density scores than carriers for all cortical areas except the hippocampal area where carriers had slightly more "frequent" scores than non-carriers. The latter was not statistically different. For tangle densities in the $\geq 90$ age group, ApoE\&4 carriers had significantly more "frequent" scores for the frontal, temporal, and parietal areas, but significantly fewer "frequent scores" for the hippocampal and entorhinal areas.

When analyzed by individual ApoE genotype, cases containing at least $1 \& 4$ allele (the $2 / 4,3 / 4$, and $4 / 4$ groups) had greater frequencies of "frequent" ratings for both plaque and tangle scores when compared to the $2 / 3$ and $3 / 3$ groups. Within the ApoE $\varepsilon 4$ carriers, the proportion of "frequent" ratings tended to increase $(2 / 4<3 / 4$
$<4 / 4)$. For all ApoE genotypes, the distribution of plaque pathology scores was skewed toward the "moderate" and "frequent" ratings for the frontal, temporal, and parietal areas (Table 5). For the entorhinal and hippocampal areas, there was a more even distribution of plaque scores among the ApoE genotypes. All groupwise comparisons showed statistically significant differences $(\mathrm{p}<0.001)$ with all $\varepsilon 4$ carriers having greater pathology than non-carriers and for increased pathology within the $\varepsilon 4$ carrier group $(2 / 4<3 / 4<4 / 4)$.

For tangle scores, the overall distribution of score frequencies was somewhat more even than plaque scores (Table 6); however the ApoE $\varepsilon 4$ carries tended to show greater tangle pathology than non-carriers across all cortical areas. For both plaque and tangle scores, all groupwise comparisons showed statistically significant differences $(p<0.005)$ with the exception of parietal tangle count where ApoE 3/3 and ApoE 3/4 p $=0.56$ and hippocampal tangle count where ApoE $3 / 3$ and ApoE $3 / 4 \mathrm{p}=0.98$. $\varepsilon 4$ carriers showed greater tangle pathology than non-carriers. Increased tangle pathology within the $\varepsilon 4$ carrier group was also noted $(2 / 4<3 / 4<4 / 4)$.

\section{Discussion}

In this study we found within a series of prospectively characterized and clinicopathologically confirmed $\mathrm{AD}$ cases, ApoE \&4 carriers had a significantly higher percentage of "frequent" scores for plaques and tangles when using the CERAD scale when compared to ApoE $\varepsilon 4$ non-carriers in several regions of the brain. These results imply that carrying the $\varepsilon 4$ allele increases the density of pathologies in several brain regions. Since AD is characterized by senile plaques and tangles, our study supports previous evidence demonstrating that the presence of the ApoE $\varepsilon 4$ allele is a strong contributor to increased AD pathology. However, our study also demonstrates a much more detailed continuum of AD pathology as it relates to ApoE genotype. To our knowledge, the relationship between ApoE genotype and AD pathology has not been reported in this manner before. Previous studies have reported $\mathrm{AD}$ pathology and $\mathrm{ApoE} \varepsilon 4$ associations in terms of odds ratios [5,19]; however reporting this association in terms of CERAD score frequency may provide a more detailed picture of the differences in $\mathrm{AD}$ pathology severity

Table 1 Demographic characteristics by ApoE $\varepsilon 4$ carrier status

\begin{tabular}{ccccc}
\hline & ApoE $\varepsilon$ 4 carrier & ApoE $\varepsilon$ 4 non-carrier & p-value & Effect size \\
\hline $\mathrm{n}(\%)$ & $312(55)$ & $254(45)$ & - & 0.28 \\
Gender $(\mathrm{M} / \mathrm{F})$ & $169 / 143$ & $126 / 128$ & $<0.001$ & - \\
Age at Death & $81.53(7.49)$ & $83.81(9.12)$ & 0.87 & 0.27 \\
Education & $14.52(2.45)$ & $14.56(2.91)$ & $<0.001$ & 0.01 \\
Braak Stage & $5.01(1.03)$ & $4.53(1.26)$ & 0.42 \\
\hline
\end{tabular}

Mean (sd = standard deviation). 
Table 2 Demographic characteristics by ApoE genotype

\begin{tabular}{|c|c|c|c|c|c|}
\hline & ApoE 2/3 & ApoE 3/3 & ApoE 2/4 & ApoE 3/4 & ApoE 4/4 \\
\hline n (\%) & $22(3.9)$ & $232(41)$ & $14(2.5)$ & $238(42)$ & 60 (10.6) \\
\hline Gender (M/F) & $17 / 5$ & 109/123 & $7 / 7$ & $124 / 114$ & $38 / 22$ \\
\hline Age at Death & $84.64(9.20)$ & $83.73(9.13)$ & $84.36(7.50)$ & $81.63(7.51)$ & $80.48(7.34)$ \\
\hline Education & $15.36(3.37)$ & $14.49(2.86)$ & $13.55(2.73)$ & $14.51(2.41)$ & $14.91(2.52)$ \\
\hline Braak Stage & $4.36(1.43)$ & $4.55(1.25)$ & 4.54 (1.39) & 4.99 (1.03) & $5.19(0.88)$ \\
\hline
\end{tabular}

Mean (sd = standard deviation).

between ApoE $\varepsilon 4$ carriers and non-carriers. Additionally, we report this association as it relates to the distribution of CERAD pathology scores among different brain regions. In both ApoE $\varepsilon 4$ carriers and non-carriers, we found that "sparse" and "moderate" plaque density scores were more prevalent in the hippocampus entorhinal regions when compared to the frontal, temporal, and parietal, regions in which "frequent" scores were more prevalent. Tangle density scores in the frontal, temporal, and parietal regions were more evenly distributed than plaque density scores within the ApoE $\varepsilon 4$ carrier and non-carrier groups; however ApoE $\varepsilon 4$ carriers had a higher prevalence of "frequent" scores for all brain regions when compared to noncarriers, except in the hippocampal and entorhinal areas among individuals age 90 and older where non-carriers had greater tangle pathology than carriers.

When analyzed by individual ApoE genotype, "none", "sparse" and "moderate" plaque density scores were more prevalent among $3 / 3$ carriers with $3 / 4$ and $4 / 4$ carriers having a higher prevalence of "frequent" scores among

Table 3 Frequency of plaque pathology scores for ApoE $\varepsilon 4$ carriers and non-carriers by age group

\begin{tabular}{|c|c|c|c|c|c|c|c|c|c|}
\hline \multirow[t]{2}{*}{ Age group } & & \multicolumn{2}{|c|}{$\leq 69$} & \multicolumn{2}{|c|}{$70-79$} & \multicolumn{2}{|c|}{$80-89$} & \multicolumn{2}{|c|}{$\geq \mathbf{9 0}$} \\
\hline & & $\varepsilon 4+$ & $\varepsilon 4$ - & $\varepsilon 4+$ & $\varepsilon 4$ - & $\varepsilon 4+$ & $\varepsilon 4$ - & $\varepsilon 4+$ & $\varepsilon 4$ - \\
\hline \multirow{5}{*}{$\begin{array}{l}\text { Frontal } \\
\text { Plaque } \\
\text { Density }\end{array}$} & None & $0(0 \%)$ & $0(0 \%)$ & $0(0 \%)$ & $0(0 \%)$ & $0(0 \%)$ & $0(0 \%)$ & $0(0 \%)$ & 1 (1\%) \\
\hline & Sparse & $0(0 \%)$ & $0(0 \%)$ & $1(0.7 \%)$ & $1(0.7 \%)$ & $0(0 \%)$ & $3(1.1 \%)$ & $0(0 \%)$ & $1(1 \%)$ \\
\hline & Moderate & $2(5.1 \%)$ & $1(2.6 \%)$ & $5(3.6 \%)$ & $8(5.8 \%)$ & $5(1.8 \%)$ & $13(4.6 \%)$ & $2(2 \%)$ & $11(10.8 \%)$ \\
\hline & Frequent & 21 (58.9\%) & 15 (38.5\%) & 86 (61.87\%) & 38 (27.3\%) & 147 (52.5\%) & 112 (40\%) & 38 (37.3\%) & 49 (48\%) \\
\hline & & \multicolumn{2}{|c|}{$p=0.78$} & \multicolumn{2}{|c|}{$p=0.03$} & \multicolumn{2}{|c|}{$p=0.003$} & \multicolumn{2}{|c|}{$p=0.03$} \\
\hline \multirow{5}{*}{$\begin{array}{l}\text { Temporal } \\
\text { Plaque } \\
\text { Density }\end{array}$} & None & $0(0 \%)$ & $0(0 \%)$ & $0(0 \%)$ & $0(0 \%)$ & $0(0 \%)$ & $0(0 \%)$ & $0(0 \%)$ & $0(0 \%)$ \\
\hline & Sparse & $0(0 \%)$ & $0(0 \%)$ & $0(0 \%)$ & $2(1.4 \%)$ & $0(0 \%)$ & $3(1.1 \%)$ & $0(0 \%)$ & $2(2 \%)$ \\
\hline & Moderate & $1(2.6 \%)$ & $0(0 \%)$ & $7(5.0 \%)$ & $6(4.3 \%)$ & $7(2.5 \%)$ & $21(7.5 \%)$ & $2(2 \%)$ & $8(7.9 \%)$ \\
\hline & Frequent & $22(56.4 \%)$ & $16(41.0 \%)$ & $85(61.2 \%)$ & 39 (28.1\%) & $146(52.1 \%)$ & 103 (36.8\%) & 38 (37.6\%) & $51(50.5 \%)$ \\
\hline & & \multicolumn{2}{|c|}{$p=0.40$} & \multicolumn{2}{|c|}{$p=0.08$} & \multicolumn{2}{|c|}{$p<0.001$} & \multicolumn{2}{|c|}{$p=0.08$} \\
\hline \multirow{5}{*}{$\begin{array}{l}\text { Parietal } \\
\text { Plaque } \\
\text { Density }\end{array}$} & None & $0(0 \%)$ & $0(0 \%)$ & $0(0 \%)$ & $0(0 \%)$ & $0(0 \%)$ & $0(0 \%)$ & $0(0 \%)$ & $2(2 \%)$ \\
\hline & Sparse & $0(0 \%)$ & $0(0 \%)$ & $0(0 \%)$ & $1(0.7 \%)$ & $0(0 \%)$ & $1(0.4 \%)$ & $0(0 \%)$ & 1 (1\%) \\
\hline & Moderate & $0(0 \%)$ & $0(0 \%)$ & $5(3.6 \%)$ & $6(4.4 \%)$ & $3(1.1 \%)$ & $14(5 \%)$ & 1 (1\%) & 7 (7\%) \\
\hline & Frequent & $23(59 \%)$ & $16(41 \%)$ & 87 (63\%) & 39 (28.3\%) & $148(53.1 \%)$ & $113(40.5 \%)$ & 39 (39\%) & $50(50 \%)$ \\
\hline & & \multicolumn{2}{|c|}{$p=0.99$} & \multicolumn{2}{|c|}{$p=0.05$} & \multicolumn{2}{|c|}{$p=0.001$} & \multicolumn{2}{|c|}{$p=0.03$} \\
\hline \multirow{5}{*}{$\begin{array}{l}\text { Hippocampal } \\
\text { Plaque } \\
\text { Density }\end{array}$} & None & $0(0 \%)$ & $0(0 \%)$ & $2(1.5 \%)$ & $7(5.1 \%)$ & $3(1.1 \%)$ & 11 (3.9\%) & $0(0 \%)$ & $8(8 \%)$ \\
\hline & Sparse & $3(7.7 \%)$ & $1(2.6 \%)$ & $5(3.6 \%)$ & $6(4.4 \%)$ & $18(6.4 \%)$ & $38(13.6 \%)$ & $9(9 \%)$ & $15(15 \%)$ \\
\hline & Moderate & $4(10.3 \%)$ & $6(15.4 \%)$ & 44 (31.9\%) & 18 (13\%) & 70 (25\%) & 41 (14.6\%) & 15 (15\%) & $23(23 \%)$ \\
\hline & Frequent & 16 (41\%) & $9(23.1 \%)$ & 40 (29\%) & 16 (11.6\%) & $62(22.1 \%)$ & 37 (13.2\%) & $16(16 \%)$ & 14 (14\%) \\
\hline & & \multicolumn{2}{|c|}{$p=0.56$} & \multicolumn{2}{|c|}{$p=0.03$} & \multicolumn{2}{|c|}{$p<0.001$} & \multicolumn{2}{|c|}{$p=0.02$} \\
\hline \multirow{5}{*}{$\begin{array}{l}\text { Entorhinal } \\
\text { Plaque } \\
\text { Density }\end{array}$} & None & $0(0 \%)$ & $0(0 \%)$ & $1(0.7 \%)$ & 4 (2.9\%) & $2(0.7 \%)$ & $2(0.7 \%)$ & $1(1 \%)$ & $3(3 \%)$ \\
\hline & Sparse & $0(0 \%)$ & $0(0 \%)$ & $1(0.7 \%)$ & $5(3.6 \%)$ & $3(1.1 \%)$ & $13(4.6 \%)$ & 1 (1\%) & $1(1 \%)$ \\
\hline & Moderate & $2(5.1 \%)$ & $2(5.1 \%)$ & $22(15.8 \%)$ & 14 (10.1\%) & 38 (13.6\%) & 39 (13.9\%) & $9(8.9 \%)$ & $24(23.8 \%)$ \\
\hline & Frequent & $21(53.9 \%)$ & 14 (35.9\%) & 68 (48.9\%) & $24(17.3 \%)$ & 110 (39.3\%) & $73(26.1 \%)$ & $29(28.7 \%)$ & 33 (32.7\%) \\
\hline & & \multicolumn{2}{|c|}{$p=0.70$} & \multicolumn{2}{|c|}{$p=0.002$} & \multicolumn{2}{|c|}{$p=0.005$} & \multicolumn{2}{|c|}{$p=0.08$} \\
\hline
\end{tabular}


Table 4 Frequency of tangle pathology scores for ApoE $\varepsilon 4$ carriers and non-carriers by age group

\begin{tabular}{|c|c|c|c|c|c|c|c|c|c|}
\hline \multirow[t]{2}{*}{ Age group } & & \multicolumn{2}{|c|}{$\leq 69$} & \multicolumn{2}{|c|}{$70-79$} & \multicolumn{2}{|c|}{$80-89$} & \multicolumn{2}{|c|}{$\geq 90$} \\
\hline & & $\varepsilon 4+$ & $\varepsilon 4$ - & $\varepsilon 4+$ & $\varepsilon 4-$ & $\varepsilon 4+$ & $\varepsilon 4$ - & $\varepsilon 4+$ & $\varepsilon 4$ - \\
\hline \multirow{5}{*}{$\begin{array}{l}\text { Frontal } \\
\text { Tangle } \\
\text { Density }\end{array}$} & None & 1 (2.6\%) & $0(0 \%)$ & $14(10.2 \%)$ & $11(8 \%)$ & 20 (7.1\%) & 36 (12.9\%) & $6(5.9 \%)$ & 19 (18.6\%) \\
\hline & Sparse & $1(2.6 \%)$ & $0(0 \%)$ & 13 (9.4\%) & $8(6 \%)$ & 31 (11.1\%) & 36 (12.9\%) & 10 (9.8\%) & $21(20.6 \%)$ \\
\hline & Moderate & $0(0 \%)$ & $0(0 \%)$ & $8(5.8 \%)$ & $5(3.6 \%)$ & 27 (9.6\%) & 10 (3.6\%) & $6(5.9 \%)$ & 10 (9.8\%) \\
\hline & Frequent & 21 (53.9\%) & $16(41.3 \%)$ & 56 (40.9\%) & $23(16.7 \%)$ & 74 (26.4\%) & $46(16.4 \%)$ & 18 (17.7\%) & 12 (11.8\%) \\
\hline & & \multicolumn{2}{|c|}{$p=0.23$} & \multicolumn{2}{|c|}{$p=0.14$} & \multicolumn{2}{|c|}{$p=0.001$} & \multicolumn{2}{|c|}{$p=0.006$} \\
\hline \multirow{5}{*}{$\begin{array}{l}\text { Temporal } \\
\text { Tangle } \\
\text { Density }\end{array}$} & None & $1(2.6 \%)$ & $0(0 \%)$ & $8(5.8 \%)$ & $8(5.8 \%)$ & 9 (3.2\%) & $13(4.6 \%)$ & $1(1 \%)$ & 8 (7.9\%) \\
\hline & Sparse & $1(2.6 \%)$ & $0(0 \%)$ & 9 (6.5\%) & 7 (5.1\%) & 12 (4.3\%) & $30(10.7 \%)$ & 9 (8.9\%) & 15 (14.9\%) \\
\hline & Moderate & $0(0 \%)$ & $0(0 \%)$ & 10 (7.3\%) & 4 (2.9\%) & $14(5 \%)$ & 20 (7.1\%) & $3(3 \%)$ & 12 (11.9\%) \\
\hline & Frequent & 21 (53.9\%) & $16(41.3 \%)$ & $64(46.4 \%)$ & $28(20.3 \%)$ & 118 (42.1\%) & 64 (22.9\%) & 27 (26.7\%) & $26(25.7 \%)$ \\
\hline & & \multicolumn{2}{|c|}{$p=0.46$} & \multicolumn{2}{|c|}{$p=0.13$} & \multicolumn{2}{|c|}{$p<0.001$} & \multicolumn{2}{|c|}{$p=0.02$} \\
\hline \multirow{5}{*}{$\begin{array}{l}\text { Parietal } \\
\text { Tangle } \\
\text { Density }\end{array}$} & None & $2(5.1 \%)$ & $0(0 \%)$ & $16(11.7 \%)$ & 12 (8.8\%) & $18(6.5 \%)$ & $42(15.1 \%)$ & 8 (8.1\%) & $20(20.2 \%)$ \\
\hline & Sparse & $0(0 \%)$ & $0(0 \%)$ & 9 (6.6\%) & $6(4.4 \%)$ & $30(10.8 \%)$ & $25(9 \%)$ & 10 (10.1\%) & 18 (18.2\%) \\
\hline & Moderate & 1 (2.6\%) & 1 (2.6\%) & $6(4.4 \%)$ & 4 (2.9\%) & 27 (9.7\%) & 13 (4.7\%) & $6(6.1 \%)$ & 9 (9.1\%) \\
\hline & Frequent & 20 (51.3\%) & 15 (38.5\%) & $60(43.8 \%)$ & $24(17.5 \%)$ & 76 (27.2\%) & $48(17.2 \%)$ & $16(16.2 \%)$ & 12 (12.1\%) \\
\hline & & \multicolumn{2}{|c|}{$p=0.23$} & \multicolumn{2}{|c|}{$p=0.12$} & \multicolumn{2}{|c|}{$p<0.001$} & \multicolumn{2}{|c|}{$p=0.03$} \\
\hline \multirow{5}{*}{$\begin{array}{l}\text { Hippocampal } \\
\text { Tangle } \\
\text { Density }\end{array}$} & None & $1(2.6 \%)$ & $0(0 \%)$ & $3(2.2 \%)$ & $5(3.6 \%)$ & $1(0.4 \%)$ & $6(2.2 \%)$ & $0(0 \%)$ & $3(3 \%)$ \\
\hline & Sparse & 1 (2.6\%) & $0(0 \%)$ & $3(2.2 \%)$ & $8(5.8 \%)$ & 7 (2.5\%) & $11(4 \%)$ & $2(2 \%)$ & $9(9 \%)$ \\
\hline & Moderate & $0(0 \%)$ & $0(0 \%)$ & $6(4.3 \%)$ & 5 (3.6\%) & 13 (4.7\%) & $21(7.6 \%)$ & $0(0 \%)$ & $7(7 \%)$ \\
\hline & Frequent & 21 (53.9\%) & $16(41 \%)$ & $80(57.6 \%)$ & $29(20.9 \%)$ & $130(46.8 \%)$ & $89(32 \%)$ & $28(38 \%)$ & $41(41 \%)$ \\
\hline & & \multicolumn{2}{|c|}{$p=0.23$} & \multicolumn{2}{|c|}{$p<0.001$} & \multicolumn{2}{|c|}{$p=0.001$} & \multicolumn{2}{|c|}{$p=0.002$} \\
\hline \multirow{5}{*}{$\begin{array}{l}\text { Entorhinal } \\
\text { Tangle } \\
\text { Density }\end{array}$} & None & $0(0 \%)$ & $0(0 \%)$ & $1(0.7 \%)$ & $1(0.7 \%)$ & $0(0 \%)$ & $1(0.4 \%)$ & $0(0 \%)$ & $0(0 \%)$ \\
\hline & Sparse & $1(2.6 \%)$ & $0(0 \%)$ & $1(0.7 \%)$ & $4(2.8 \%)$ & $1(0.4 \%)$ & $6(2.2 \%)$ & $0(0 \%)$ & $1(1 \%)$ \\
\hline & Moderate & $1(2.6 \%)$ & $0(0 \%)$ & $5(3.6 \%)$ & $3(2.2 \%)$ & $3(1.1 \%)$ & $16(5.8 \%)$ & $1(1 \%)$ & $10(10 \%)$ \\
\hline & Frequent & $21(53.9 \%)$ & $16(41 \%)$ & $85(61.2 \%)$ & $39(28.1 \%)$ & $147(53.1 \%)$ & $103(45.5 \%)$ & $38(38 \%)$ & $50(50 \%)$ \\
\hline & & \multicolumn{2}{|c|}{$p=0.23$} & \multicolumn{2}{|c|}{$p=0.08$} & \multicolumn{2}{|c|}{$p<0.001$} & \multicolumn{2}{|c|}{$p=0.02$} \\
\hline
\end{tabular}

$\mathrm{n}(\%=$ percent $)$.

the different regions. A similar pattern was also noted for tangle density scores. There was a trend among all ApoE genotype groups for tangle pathology to be less frequent than plaque pathology in the frontal, temporal, and parietal regions. These results suggest that ApoE $\varepsilon 4$ carries and non-carriers have independent neurodegenerative pathways in which plaque and tangle frequency vary among different cortical regions. Given that ApoE $\varepsilon 4$ carrier status has been shown to result in differing clinical phenotypes of AD, [19-21] our results support these findings in demonstrating pathophysiologic differences between $\mathrm{ApoE} \varepsilon 4$ carriers and non-carriers.

Nagy et al. [1] found that, among all ApoE genotypes, 4/4 carriers displayed the greatest amount of AD pathology which is consistent with our results. In the current study, plaque and tangle pathologies increased in the genotype order $2 / 3<3 / 3<2 / 4<3 / 4<4 / 4$ which has also been previously reported $[5,6]$. Ohm et al. [2] showed that the mean stages for beta amyloid deposition and Braak stages for NFTs were higher in those who carried the ApoE $\varepsilon 4$ allele in comparison to non-carriers [2,5]. ApoE $\varepsilon 4$ carriers not only had higher Braak NFT stages, but also an accelerated development of neurofibrillary changes $[2,5]$. Additionally, those who were homozygous for the $\varepsilon 4$ allele had higher mean Braak stages than $\varepsilon 4$ heterozygotes.

Beffert et al. [11] found that subjects with AD had decreased ApoE levels in both the hippocampus and frontal cortex. However, beta amyloid levels were significantly higher in AD cases compared controls. Those with the ApoE $\varepsilon 4$ allele were found to have higher levels of $A \beta$ (1-40 and 1-42) and lower levels of ApoE compared to non-carriers of the allele. From these results, Beffert et al. [11] suggest that lower ApoE levels are associated with increased $A \beta$ levels.

Richey et al. [22] found that ApoE bound avidly to senile plaques and NFTs in AD brains, suggesting a direct interaction between $A p o E$ and the aggregation of $A \beta$ and tau. It has been suggested that increased ApoE levels may be the result of a neuroprotective mechanism that is 
Table 5 Frequency of plaque pathology scores for all ApoE genotypes

\begin{tabular}{|c|c|c|c|c|c|c|}
\hline & & $2 / 3$ & $3 / 3$ & $2 / 4$ & $3 / 4$ & $4 / 4$ \\
\hline \multirow{4}{*}{$\begin{array}{l}\text { Frontal } \\
\text { Plaque } \\
\text { Density }\end{array}$} & None & $0(0 \%)$ & $1(0.4 \%)$ & $0(0 \%)$ & $0(0 \%)$ & $0(0 \%)$ \\
\hline & Sparse & $1(4.5 \%)$ & $4(1.7 \%)$ & $0(0 \%)$ & $0(0 \%)$ & $1(0 \%)$ \\
\hline & Moderate & $5(22.7 \%)$ & $28(21.4 \%)$ & $1(7.7 \%)$ & 11 (4.7\%) & $2(3.3 \%)$ \\
\hline & Frequent & $16(72.7 \%)$ & 198 (85.7\%) & 12 (92.3\%) & 224 (95.3\%) & 57 (96.7\%) \\
\hline \multirow{4}{*}{$\begin{array}{l}\text { Temporal } \\
\text { Plaque } \\
\text { Density }\end{array}$} & None & $0(0 \%)$ & $0(0 \%)$ & $0(0 \%)$ & $0(0 \%)$ & $0(0 \%)$ \\
\hline & Sparse & $1(4.5 \%)$ & $6(2.6 \%)$ & $0(0 \%)$ & $0(0 \%)$ & $0(0 \%)$ \\
\hline & Moderate & $5(22.7 \%)$ & 30 (13.1\%) & 1 (7.7\%) & 15 (6.4\%) & 1 (1.7\%) \\
\hline & Frequent & 16 (72.7\%) & 193 (84.3\%) & 12 (92.3\%) & $221(93.6 \%)$ & 59 (98.3\%) \\
\hline \multirow{4}{*}{$\begin{array}{l}\text { Parietal } \\
\text { Plaque } \\
\text { Density }\end{array}$} & None & $0(0 \%)$ & $2(0.9 \%)$ & $0(0 \%)$ & $0(0 \%)$ & $0(0 \%)$ \\
\hline & Sparse & $0(0 \%)$ & $3(1.3 \%)$ & $0(0 \%)$ & $0(0 \%)$ & $0(0 \%)$ \\
\hline & Moderate & $5(22.7 \%)$ & $22(9.6 \%)$ & $0(0 \%)$ & $6(3 \%)$ & $3(5 \%)$ \\
\hline & Frequent & $17(77.3 \%)$ & $201(88.2 \%)$ & 13 (100\%) & 229 (97\%) & 56 (95\%) \\
\hline \multirow{4}{*}{$\begin{array}{l}\text { Hippocampal } \\
\text { Plaque } \\
\text { Density }\end{array}$} & None & $6(27.3 \%)$ & $20(8.8 \%)$ & 1 (7.7\%) & $4(1.7 \%)$ & $0(0 \%)$ \\
\hline & Sparse & $6(27.3 \%)$ & $54(23.7 \%)$ & $3(23.1 \%)$ & $28(11.9 \%)$ & $5(8.3 \%)$ \\
\hline & Moderate & 7 (31.8\%) & 81 (35.5\%) & $3(23.1 \%)$ & $108(46 \%)$ & $22(36.7 \%)$ \\
\hline & Frequent & $3(13.6 \%)$ & 73 (32\%) & $6(46.1 \%)$ & 95 (40.4\%) & $33(55 \%)$ \\
\hline \multirow{4}{*}{$\begin{array}{l}\text { Entorhinal } \\
\text { Plaque } \\
\text { Density }\end{array}$} & None & 1 (4.5\%) & $8(3.5 \%)$ & $0(0 \%)$ & $3(1.3 \%)$ & $1(1.7 \%)$ \\
\hline & Sparse & $5(22.7 \%)$ & 14 (6.1\%) & $2(15.4 \%)$ & $3(1.3 \%)$ & $0(0 \%)$ \\
\hline & Moderate & 4 (18.2\%) & 75 (32.8\%) & $2(15.4 \%)$ & 59 (25\%) & 10 (16.7\%) \\
\hline & Frequent & $12(54.5 \%)$ & $132(57.6 \%)$ & 9 (69.2\%) & 171 (72.4\%) & 49 (81.7\%) \\
\hline
\end{tabular}

triggered in response to the formation of NFTs [23]. If ApoE does play a protective role, then its common isoforms and their effects could vary in efficiency, with ApoE $\varepsilon 4$ being the least efficient and $\varepsilon 2$ the most. Beffert et al. [11] found decreased levels of ApoE in the hippocampus and the frontal cortex of $\mathrm{AD}$ brains which suggests that lower ApoE levels may make the brain more susceptible to the aggregation of $\mathrm{AD}$ pathology.

However, a study by Wisniewski et al. [24] showed that $A \beta$ increases area ssociated with ApoE $\varepsilon 3$ and $\varepsilon 4$ in comparison to $A \beta$ alone,with the $\varepsilon 4$ isoform having the highest rate of increase in $A \beta$ production. This implicates ApoE's role as an accelerator for $A \beta$ formation. It was also shown in vitro that the carboxyl-terminus of ApoE could, itself, form amyloid-like fibrils, which were congo-red positive and are present in senile plaques, further emphasizing its role as a pathological chaperone [25]. Holtzman et al. [26] highlight the importance of ApoE lipidation status as it relates to amyloid deposition stating that decreases in ApoE lipidation lead to increased amyloid deposition through increased fibrillization of $A \beta$. In particular, $A p o E \varepsilon 4$ is associated with greater $A \beta$ fibrillization. Jiang et al. [27] state that ApoE lipidation status is important in terms of determining whether $A \beta$ peptides are cleared from the brain or whether they fibrillize and become amyloid deposits.
Guo et al. [28] investigated the role of $A \beta$ and its role as a potential neuroinflammatory stimulator in $\mathrm{AD}$. The study found that ApoE $\varepsilon 3$ and ApoE $\varepsilon 4$ suppressed $A \beta$ induced endogenous ApoE levels, with ApoE $\varepsilon 4$ having a more effective inhibitory action. However, it was shown that in the absence of $A \beta$, both of these ApoE isoforms stimulated cytokine interleukin-1 $\beta$ (IL-1 $\beta$ ), a pro-inflammatory agent. Specifically, ApoE $\varepsilon 4$ was associated with significantly more production of IL- $1 \beta$ than ApoE $\varepsilon 3$. Guo et al. [28] concluded that overproduction of ApoE may trigger this particular pro-inflammatory response. It is also suggested that ApoE $\varepsilon 4$ could be a less effective antiinflammatory isoform compared to $\varepsilon 2$ and $\varepsilon 3$, explaining its association with higher risk for $\mathrm{AD}$ [29]. Others have suggested that ApoE $\varepsilon 4$ is associated with increased $A \beta$ deposition and compromised neural repair mechanisms which, in conjunction, are associated with increased risk and observed pathology in AD [30].

One positive aspect of this study is the large sample size which allowed for differences to be seen between the different ApoE genotypes. Although the CERAD scoring scheme is commonly used to quantify $\mathrm{AD}$ pathology, it uses a semi-quantitative scoring system and does not provide an exact measurement of pathological densities. One weakness of our study was the low number of ApoE 
Table 6 Frequency of tangle pathology scores for all ApoE genotypes

\begin{tabular}{|c|c|c|c|c|c|c|}
\hline & & $2 / 3$ & $3 / 3$ & $2 / 4$ & $3 / 4$ & $4 / 4$ \\
\hline \multirow{4}{*}{$\begin{array}{l}\text { Frontal } \\
\text { Tangle } \\
\text { Density }\end{array}$} & None & $9(4.1 \%)$ & 57 (24.7\%) & 2 (15.4\%) & 35 (15\%) & $4(6.7 \%)$ \\
\hline & Sparse & $3(13.6 \%)$ & $62(26.8 \%)$ & $4(26.7 \%)$ & 39 (16.7\%) & $12(20 \%)$ \\
\hline & Moderate & $3(13.6 \%)$ & 22 (9.5\%) & $2(15.4 \%)$ & $31(5.6 \%)$ & 8 (13.3\%) \\
\hline & Frequent & 7 (31.8\%) & 90 (39\%) & $5(38.5 \%)$ & $129(55.1 \%)$ & 36 (60\%) \\
\hline \multirow{4}{*}{$\begin{array}{l}\text { Temporal } \\
\text { Tangle } \\
\text { Density }\end{array}$} & None & 4 (18.2\%) & 25 (10.9\%) & $2(15.4 \%)$ & 17 (7.2\%) & $0(0 \%)$ \\
\hline & Sparse & $6(27.3 \%)$ & 46 (20.1\%) & $2(15.4 \%)$ & 22 (9.4\%) & $7(11.7 \%)$ \\
\hline & Moderate & $2(9 \%)$ & 34 (14.8\%) & 1 (7.7\%) & 19 (8.1\%) & 7 (11.7\%) \\
\hline & Frequent & $10(45.5 \%)$ & $124(54.1 \%)$ & $8(61.5 \%)$ & $177(75.3 \%)$ & 46 (76.7\%) \\
\hline \multirow{4}{*}{$\begin{array}{l}\text { Parietal } \\
\text { Tangle } \\
\text { Density }\end{array}$} & None & $10(45.5 \%)$ & 64 (28.2\%) & $3(23.1)$ & 36 (15.4\%) & $5(8.5 \%)$ \\
\hline & Sparse & $2(9 \%)$ & 47 (20.7\%) & $3(23.1)$ & $36(15.4 \%)$ & 10 (5.9\%) \\
\hline & Moderate & $2(9 \%)$ & 25 (11\%) & $1(7.7 \%)$ & 31 (13.2\%) & $8(13.6 \%)$ \\
\hline & Frequent & 8 (36.4\%) & 91 (40.1\%) & $6(46.1 \%)$ & 131 (56\%) & 36 (61\%) \\
\hline \multirow{4}{*}{$\begin{array}{l}\text { Hippocampal } \\
\text { Tangle } \\
\text { Density }\end{array}$} & None & $3(13.6 \%)$ & 11 (4.8\%) & 1 (8.3\%) & $4(1.7 \%)$ & $0(0 \%)$ \\
\hline & Sparse & $1(4 \%)$ & $27(11.8 \%)$ & $0(0 \%)$ & 9 (3.8\%) & $4(6.7 \%)$ \\
\hline & Moderate & $3(13.6 \%)$ & 30 (13.2\%) & 1 (8.3\%) & 17 (7.2\%) & $2(3 \%)$ \\
\hline & Frequent & 15 (36.4\%) & $160(70.2 \%)$ & $10(83.3 \%)$ & 205 (87.2\%) & 54 (90\%) \\
\hline \multirow{4}{*}{$\begin{array}{l}\text { Entorhinal } \\
\text { Tangle } \\
\text { Density }\end{array}$} & None & 1 (13.6\%) & $11(4.8 \%)$ & 1 (7.7\%) & $0(0 \%)$ & $0(0 \%)$ \\
\hline & Sparse & $1(4.5 \%)$ & $27(11.8 \%)$ & $0(0 \%)$ & $3(1.3 \%)$ & $0(0 \%)$ \\
\hline & Moderate & 1 (13.6\%) & 30 (13.2\%) & $1(7.7 \%)$ & 7 (3.0\%) & 2 (3.3\%) \\
\hline & Frequent & $19(0 \%)$ & 160 (70.2\%) & 11 (84.6\%) & 223 (95.7\%) & 58 (96.7\%) \\
\hline
\end{tabular}

$\mathrm{n}(\%=$ percent).

$2 / 4$ carriers relative to the other genotypes so the comparisons of pathology score frequencies to the other genotypes may be somewhat biased. However, since the estimated prevalence of the ApoE 2/4 isoform is relatively low (2\%) [4] and since the $\varepsilon 2$ allele confers the lowest risk of developing $\mathrm{AD}$ [4], it would appear to be difficult to obtain a large number of these cases. The low prevalence of $\varepsilon 2$ carriers in the general population may also be the reason that no ApoE 2/2 carriers were present in the sample as previous studies have estimated $\varepsilon 2$ prevalence at approximately 7 to 8 percent [14,31]. Also, the prevalence of ApoE $\varepsilon 4$ carriers in this study was substantially higher than what would be expected in the general population [14]. A meta analysis conducted by Ward et al. [32] found that the pooled prevalence rate of ApoE $\varepsilon 4$ carriers in $\mathrm{AD}$ studies was $48.7 \%$ (95\% CI: 46.5\% - 51.0\%). This suggests that AD studies are likely to have higher proportions of ApoE $\varepsilon 4$ carriers relative to the general population given the role of ApoE $\varepsilon 4$ as a risk factor for AD. From these results, it is not surprising that our sample also had a high proportion of ApoE \&4 carriers.

Another point of consideration is whether or not these results are dependent upon the proportion of different clinicopathologic subtypes of AD. Murray et al. [19] report that up to $25 \%$ of pathologically confirmed AD cases may be those with atypical pathologic presentations (limbic predominant [LP], hippocampal sparing [HpSp]).
This study also found that the LP and HpSp subtypes were associated with ApoE $\varepsilon 4$ non-carrier status, however this association was not statistically significant.

Also, the geographical area from which tissue samples were collected is relatively homogenous with respect to ethnicity and socioeconomic status so it cannot be stated that these results would apply to populations that are more ethnically and intellectually diverse.

\section{Conclusion}

The results of this study demonstrate that the density and cortical distribution of AD neuropathology differs between ApoE $\varepsilon 4$ carriers and non-carriers. It is possible that these neuropathologic differences may play a role in determining clinical phenotype of AD. These results also suggest that different disease pathways are associated with ApoEc4 carrier status resulting in different distributions of AD neuropathology.

\section{Competing interests}

The authors have no competing interests to disclose.

\section{Authors' contributions}

MNS participated in the study design, performed clinical assessments, made clinical diagnoses, and drafted the manuscript. MM participated in the study design, drafted the manuscript, and carried out the statistical analyses. BND drafted the manuscript and acquired tissue samples. $\mathrm{KL}$ participated in the study design and drafted the manuscript. LIS drafted the manuscript and acquired tissue samples. GS drafted the manuscript and acquired tissue samples. DGW performed ApoE genotyping and drafted the manuscript. KD 
performed clinical assessments and drafted the manuscript. SAJ performed clinical assessments, made clinical diagnoses, drafted the manuscript. TGB drafted the manuscript and acquired tissue samples. All authors read and approved the final manuscript.

\section{Acknowledgements}

We are grateful to the Banner Sun Health Research Institute Brain and Body Donation Program of Sun City, Arizona for the provision of brain tissue for this study. The Brain and Body Donation Program is supported by the National Institute of Neurological Disorders and Stroke (U24 NS072026 National Brain and Tissue Resource for Parkinson's Disease and Related Disorders), the National Institute on Aging (P30 AG19610 Arizona Alzheimer's Disease Core Center), the Arizona Department of Health Services (contract 211002, Arizona Alzheimer's Research Center), the Arizona Biomedical Research Commission (contracts 4001, 0011, 05-901 and 1001 to the Arizona Parkinson's Disease Consortium) and the Michael J. Fox Foundation for Parkinson's Research.

\section{Author details}

${ }^{1}$ The Cleo Robert Center for Clinical Research, Banner Sun Health Research Institute, Sun City, AZ, USA. ${ }^{2}$ Civin Laboratory for Neuropathology, Banner Sun Health Research Institute, Sun City, AZ, USA. ${ }^{3}$ Laboratory of Neuroinflammation, Banner Sun Health Research Institute, Sun City, AZ, USA

Received: 5 October 2012 Accepted: 3 May 2013

Published: 11 May 2013

\section{References}

1. Nagy Z, Esirir MM, Jobst KA, Johnston C, Litchfield S, Sim E, Smith AD: Influence of the Apolipoprotein E genotype on amyloid deposition and neurofibrillary tangle formation in Alzheimer's disease. Neuroscience 1995, 69(3):757-761.

2. Ohm TG, Kirca M, Bohl J, Scharnagl H, Grob W, Marz W: Apolipoprotein E polymorphism influences not only cerebral senile plaque load but also Alzheimer-type neurofibrillary tangle formation. Neuroscience 1995, 66(3):583-587

3. Caselli RJ, Walker D, Sue L, Sabbagh M, Beach T: Amyloid load in nondemented brains correlates with APOE e4. Neurosci Lett 2010, 473(3):168-171.

4. Raber J, Huang Y, Ashford JW: ApoE genotype accounts for the vast majority of AD risk and AD pathology. Neurobiol Aging 2004, 25(5):641-650.

5. Corder E, Saunders A, Strittmatter W, Schmechel D, Gaskell P, Small G, Roses A, Haines J, Pericak-Vance M: Gene dose of Apolipoprotein E type 4 allele and the risk of Alzheimer's disease in late onset families. Science 1993, 261(5123):921-923.

6. DeMattos RB: Apolipoprotein E dose-dependent modulation of $\beta$-amyloid deposition in a transgenic mouse model of Alzheimer's disease. J Mol Neurosci 2004, 23(3):255-262.

7. Crutcher KA: Apolipoprotein E is a prime suspect, not just an accomplice, in Alzheimer's disease. J Mol Neurosci 2004, 23(3):181-188.

8. Verghese PB, Castellano JM, Holtzman DM: Apolipoprotein E in Alzheimer's disease and other neurological disorders. Lancet Neurol 2011, 10(3):241-252.

9. Fullerton SM, Clark AG, Weiss KM, Nickerson DA, Taylor SL, Stengard JH, Salomaa V, Vartiainen E, Perola M, Boerwinkle E, Sing CF: Apolipoprotein E variation at the sequence haplotype level: implications for the origin and maintenance of a major human polymorphism. Am J Hum Genet 2000, 67(4):881-900.

10. Saunders AM, Strittmatter WJ, Schmechal D, George-Hyslop PH, PericakVance MA, Joo SH, Rosi BL, Gusella JF, Crapper-MacLachlan DR, Alberts MJ, Hulette C, Crain B, Goldgaber D, Roses AD: Association of Apolipoprotein E allele $\varepsilon 4$ with late-onset familial and sporadic Alzheimer's disease. Neurology 1993, 43(8):1467-1472.

11. Beffert U, Cohn JS, Petit-Turcotte C, Tremblay M, Aumont N, Ramassamy C, Davignon J, Poirier J: Apolipoprotein $E$ and $\beta$-amyloid Levels in the hippocampus and frontal cortex of Alzheimer's disease subjects are disease-related and Apolipoprotein E genotype dependent. Brain Res 1999, 843(1-2):87-94.

12. Thal DR, Udo R, Orantes M, Braak H: Phases of $A \beta$-deposition in the human brain and its relevance for the development of AD. Neurology 2002, 58(12):1791-1800.

13. Braak H, Braak E: Neuropathological stageing of Alzheimer-related changes. Acta Neuropathol 1991, 82(4):239-259.
14. Beach TG, Sue $L I$, Walker DG, et al: The Sun health research institute brain donation program: description and experience, 1987-2007. Cell Tissue Bank 2008, 9(3):229-245.

15. American Psychiatric Association: Diagnostic and statistical manual of mental disorders. 4th edition. Washington DC: American Psychiatric Press; 1987.

16. The National Institute on aging, and Reagan Institute Working Group on diagnostic criteria for the Neuropathological Assessment of Alzheimer's Disease: Consensus recommendations for the postmortem diagnosis of Alzheimer's disease. Neurobiol Aging 1997, 18(4 Suppl):S1-S2.

17. Mirra SS, Heyman A, McKeel D, Sumi SM, Crain BJ, Brownlee LM, Vogel FS, Hughes JP, van Belle G, Berg L: The consortium to establish a registry for Alzheimer's disease (CERAD). part II. Standardization of the neuropathologic assessment of Alzheimer's disease. Neurology 1991 41(4):479-486

18. Hixson JE, Vernier DT: Restriction isotyping of human apolipoprotein $E$ by gene amplification and cleavage with Hhal. J Lipid Res 1990, 31(3):545-548.

19. Murray ME, Graff-Radford N, Ross OA, Petersen RC, Duara R, Dickson DW: Neuropathologically defined subtypes of Alzheimer's disease with distinct clinical characteristics: a retrospective study. Lancet Neurol 2011, 10(9):785-796

20. Dickerson BC, Wolk DA, the Alzheimer's Disease Neuroimaging Initiative: Dysexecutive versus amnesic phenotypes of very mild Alzheimer's disease are associated with distinct clinical, genetic and cortical thinning characteristics. J Neurol Neurosurg Psychiatry 2011, 82(1):45-51.

21. van der Flier WM, Schoonenboom SWM, Pijnenburg YAL, Fox NC, Sheltens $P$ : The effect of APOE genotype on clinical phenotype in Alzheimer disease. Neurology 2006, 67(3):526-527.

22. PI R, Siedlak SI, Smith MA, Perry G: Apolipoprotein E interaction with the neurofibrillary tangles and senile plaques in Alzheimer disease: implications for disease pathogenesis. Biochem Biophys Res Commun 1995, 208(2):657-663.

23. Ignatius MJ, Gebicke-Harter PJ, Skene JHP, Schilling JW, Weisgraber KH, Mahley RW, Shooter EM: Expression of Apolipoprotein E during nerve degeneration and regeneration. Proc Natl Acad Sci USA 1986, 83(4):1125-1129.

24. Wisniewski T, Castano EM, Golabek A, Vogel T, Frangione B: Acceleration of Alzheimer's fibril formation by Apolipoprotein $\mathrm{E}$ in vitro. Am J Pathol 1994, 145(5):1030-1035.

25. Wisniewski T, Lalowski M, Golabek B, Frangione B, Vogel T: Is Alzheimer's disease an Apolipoprotein E amyloidosis? Lancet 1995, 345(8955):956-958.

26. Holtzman DM, Herz J, Bu G: Apolipoprotein E and apolipoprotein E receptors: normal biology and roles in Alzheimer disease. Cold Spring Harb Perspect Med 2012, 2(3):a006312.

27. Jiang Q, Lee CYD, Mandrekar S, Wilkinson B, Cramer P, Zelcer N, Mann K, Lamb B, Willson TM, Collins JL, Richardson JC, Smith JD, Comery TA, Riddell D, Holtzman DM, Tonotonoz P, Landreth GE: ApoE promotes the proteolytic degradation of A . Neuron 2008, 58(5):681-693.

28. Guo L, LaDu MJ, Van Eldik LJ: A dual role for apolipoprotein $E$ in neuroinflammation: anti- and pro-inflammatory activity. J Mol Neurosci 2004, 23(3):205-212.

29. Griffin WST, Sheng JG, Royston MC, Gentleman SM, McKenzie JE, Graham DI, Roberts GW, Mrak RE: Glial-neuronal interactions in Alzheimer's disease: the potential role of a 'cytokine cycle' in disease progression. Brain Pathol 1998, 8(1):65-72

30. Baum L, Chen L, Nq HK, Pang CP: Apolipoprotein E isoforms in Alzheimer's disease pathology and etiology. Microsc Res Tech 2000, 50(4):278-281

31. Schiefermeier M, Kollegger H, Madl C, Schwarz C, Holzer M, Kofler J, Sterz F: Apolipoprotein E polymorphism: survival and neurological outcome after cardiopulmonary resuscitation. Stroke 2000, 31(9):2068-2073.

32. Ward A, Crean S, Mercaldi CJ, Collins JM, Boyd D, Cook MN, Arrighi HM: Prevalence of apolipoprotein e4 genotype and homozygotes (APOE e4/4) among patients diagnosed with Alzheimer's disease: a systematic review and meta-analysis. Neuroepidemiology 2012, 38(1):1-17.

doi:10.1186/1471-2377-13-44

Cite this article as: Sabbagh et al:: The influence of Apolipoprotein $\mathrm{E}$ genotype on regional pathology in Alzheimer's disease. BMC Neurology 2013 13:44 